\title{
Increase in Infectious Diseases as a Result of Poor Environmental Sanitation in the Tamale Metropolis
}

\author{
Dr. Abdallah Iddrisu Yahaya* \\ Senior Lecturer, University for Development Studies, UDS, Dept. Of Medicine and Allied Science, Clinician, \\ Tamale Teaching Hospital, Regional Tb Clinician, Tamale, Head Sof Chest Unit, Tamale Teaching Hospital, \\ Ghana \\ *Corresponding Author: Dr. Abdallah Iddrisu Yahaya, Senior Lecturer, University for Development \\ Studies, UDS, Dept. Of Medicine and Allied Science, Clinician, Tamale Teaching Hospital, Regional Tb \\ Clinician, Tamale, Head Sof Chest Unit, Tamale Teaching Hospital, Ghana
}

\begin{abstract}
A retrospective review of records of patients attending Tamale Teaching Hospital (TTH), Central Hospital and West Hospital was done from 2013 to 2016 to assess the mortality and morbidity caused by communicable and non-communicable diseases burden which were of epidemic status in relation to the environment.

Further investigation showed that poor environmental sanitation is due to rapid population growth and unplanned urbanization resulting in higher disease burden.
\end{abstract}

Keywords: Retrospective, Sanitation, Unplanned Urbanisation, Infectious Diseases, Review of Records

\section{INTRODUCTION}

Adequate sanitation, together with good hygiene and safe water, are fundamental to good health and to social and economic development. That is why, in 2008, the Prime Minister of India quoted Mahatma Gandhi who said in 1923, "sanitation is more important than independence". Improvements in one or more of these three components of good health can substantially reduce the rates of morbidity and the severity of various diseases and improve the quality of life of huge numbers of people, particularly children, in developing countries. Lack of sanitation leads to disease, as was first noted scientifically in 1842 in Chadwick's seminal "Report on an inquiry into the sanitary condition of the labouring population of Great Britain". A less scientifically rigorous but nonetheless professionally significant indicator of the impact on health of poor sanitation was provided in 2007, when readers of the BMJ (British Medical Journal) voted sanitation the most important medical milestone since 1840 .

The diseases associated with poor sanitation are particularly correlated with attitude, poverty and infancy and alone account for about $10 \%$ of the global burden of disease. At any given time close to half of the urban populations of Africa, Asia, and Latin America have a disease associated with poor sanitation, hygiene, and water.

\section{BACKGROUND}

Information on the burden of disease, its causes and prevention is fundamental to health policy. Among other things, an improved understanding of the disease burden and the relative contribution of key risks points towards opportunities for preventive action in a context of increasing healthcare costs (OECD 2013).

WHO estimated the disease burden from water, sanitation, and hygiene at the global level taking into account various disease outcomes, principally diarrheal diseases. The disability-adjusted life year (DALY) combines the burden from death and disability in a single index and permits the comparison of the burden from water, sanitation, and hygiene with the burden from other risk factors or diseases. By dividing the world's population into typical exposure scenarios for 14 geographical regions, and then matching these scenarios with relative risk information obtained mainly from intervention studies, the estimated disease burden from water, sanitation, and hygiene was $4.0 \%$ of all deaths and 
$5.7 \%$ of the total disease burden (in DALYs) occurring worldwide, taking into account diarrheal diseases, schistosomiasis, trachoma, ascariasis, trichuriasis, and hookworm disease. This preliminary estimation of the global disease burden caused by water, sanitation, and hygiene provides a basic model that could be further refined for national or regional assessments.

Non-communicable diseases have been established as a clear threat not only to human health, but also to development and economic growth. Claiming $63 \%$ of all deaths, these diseases are currently the world's main killer. Eighty percent of these deaths now occur in low and middle income countries (World Economic Forum, 2010). Half of those who die of chronic non-communicable diseases are in the prime of their productive years and thus, the disability imposed and the lives lost are also endangering industry competiveness across borders.

The evidence gathered is compelling. Over the next 20 years, NCDs will cost more than US\$ 30 trillion, representing $48 \%$ of global GDP in 2010, and pushing millions of people below the poverty line. Mental health conditions alone will account for the loss of an additional US\$16.1 trillion, over this time span, with dramatic impact on productivity and quality of life.

The rise in the prevalence and significance of NCDs is the result of complex interaction between health, economic growth and development, and it is strongly associated with universal trends such as ageing of the global population, rapid unplanned urbanization and the globalization of unhealthy lifestyles. In addition to the tremendous demands that these diseases place on social welfare and health systems, they also cause decreased productivity in the workplace, prolonged disability and diminished resources within families.

The results are unequivocal: a unified front is needed to turn the tide on NCDs. Governments but also civil society and the private sector must commit to the highest level of engagement in combating these diseases and their rising economic burden. Global business leaders are acutely aware of the problems posed by NCDs. A survey of business executives from around the world conducted by the world economic forum since 2009, identified NCDs as one of the leading threats to global economic growth. Therefore, it is also important for the private sector to have a strategic vision on how to fulfil its role as a key agent for change and how to facilitate the adoption of healthier lifestyles including good sanitary and hygienic practices not only by consumers, but also by employees.

\section{RATIONAL OF THE STUDY}

It has been recognized that, as society's modernise they experience significant changes in their patterns of health and disease. Despite rapid modernization across the globe, there are relatively few detailed case studies of changes in health and disease within specific countries especially for SubSahara Africa countries.

Today, hundreds of millions of people do not have access to improved sources of drinking water, leaving them at risk for water, sanitation, and hygiene related diseases. Globally, in $2010,801,000$ children died from diarrheal illnesses, most of which are caused by unsafe water, poor sanitation, and inadequate hygiene. Devastating epidemics of cholera, such as the 2010 - 2011 outbreak in Haiti that caused more than 500,000 cases of illness and 7,000 deaths, are only the "tip of the iceberg," as most waterborne diseases, illnesses, and deaths are never reported.

Half of the people living in developing countries do not have access to even a basic toilet. This presents a major risk to public health. Diseases attributable to poor sanitation currently kill more children globally than AIDS, malaria and measles put together, and diarrhoea is the single biggest killer of children in Africa. Safe sanitation is widely acknowledged to be an essential foundation for better health, welfare and economic productivity, but progress in reducing the burden of sanitationrelated diseases borne by poor people in developing countries remains slow and is holding back progress on all other development outcomes.

This study presents evidence to illustrate the nature and speed of the epidemiological transition in relation to sanitation in the Tamale Metropolitan Area of Ghana.

\section{LITERATURE}

According to Dalals et al, the prevalence of NCDs and their risk factors is high in some SSA settings, with lack of vital statistics systems; epidemiological studies with variety of in-depth analysis of risk factors could provide a better understanding of NCD. 
$63 \%$ of all deaths worldwide currently stem from NCDs, chiefly cardiovascular disease, cancer, chronic respiratory diseases and diabetes. These deaths are distributed widely among the world's population from high income to low income countries and from young to old (about one quarter of all NCD deaths occur below the age of 60 , amounting to approximately 9 million deaths per year). NCDs have a large impact, undercutting productivity and boosting healthcare outlays. Moreover, the number of people affected by NCDs is expected to rise substantially in the coming decades, reflecting the ageing increasing global population. What were once considered "diseases of affluence" have also now encroached on developing countries.

NCDs stem from a combination of modifiable and non-modifiable risk factors. Non- modifiable risk factors refer to characteristics that cannot be changed by an individual (or the environment)and include, age, sex and genetic make-up. Although, they cannot be the primary targets of interventions, they remain important factors since they affect and partly determine the effectiveness of many prevention and treatment approaches. Modifiable risk factors include Tobacco use, Alcohol intake, Unhealthy diet and Physical inactivity including poor sanitation and hygiene practices.

Mounting evidence highlights how millions of deaths can be averted and economic losses reduced by billions of dollars if added focus is put on prevention. A recent world health organization report underlines that population based measures for reducing tobacco and harmful alcohol use, as well as unhealthy diet and physical inactivity, are estimated to cost USS 2 billion per year for all low and middle income countries, which in fact translates to less than USS 0.40 per person.

The disease burden from unsafe water, sanitation and hygiene (WSH) is usually estimated at the global level taking into account various disease out- comes, principally diarrhoeal diseases. The risk factor is defined as including multiple factors, namely the ingestion of unsafe water, lack of water linked to inadequate hygiene, poor personal and domestic hygiene and agricultural practices, contact with unsafe water, and inadequate development and management of water resources or water systems.

For estimating disease burden of infectious diarrhoea, exposure scenarios are established according to water supply and sanitation infrastructure, the level of faecal oral pathogens in the environment and populations assigned to these scenarios. The total burdens from schistosomiasis, trachoma, ascariasis, trichuriasis and hookworm disease are all wholly attributable to unsafe water, sanitation and hygiene and have been quantified at global level as an additional exercise.

Unsafe water, sanitation and hygiene is an important determinant in a number of additional diseases, such as malaria, yellow fever, filariasis, dengue, hepatitis A and hepatitis E, typhoid fever, arsenicosis, fluorosis and legionellosis, some of which present a high disease burden at global level.

For infectious diarrhoea, six exposure levels were defined, with the lowest risk level corresponding to an ideal situation where unsafe water, sanitation and hygiene plays no role in disease transmission. Exposure prevalence, in terms of infrastructure, was determined from the Global Water Supply and Sanitation Assessment 2000. This assessment is a synthesis of major international surveys and national census reports covering $89 \%$ of the global population. The parameters considered included access to improved water sources and improved sanitation facilities.

Studies have suggested ranges of reductions in diarrhoea incidence that could be achieved by reducing the transmission of faecal-oral pathogens through the implementation of interventions, such as point of use treatment and disinfection of stored water (Quick et al. 1999; Semenza et al. 1998). The disease burden from unsafe water, sanitation and hygiene was estimated to have been 1.73 million deaths in the year 2000, and $88 \%$ of the global burden of diarrhoeal disease due to infectious diarrhoeal diseases. In addition, schistosomiasis, trachoma, ascariasis, trichuriasis and hookworm disease are fully attributable to unsafe water, sanitation and hygiene related factors. Typically, the fraction of diarrhoeal disease attributed to unsafe water, sanitation and hygiene in developed countries is approximately $60 \%$, whereas in developing countries as much as $85-90 \%$ of diarrhoeal illness can be attributed to unsafe water, sanitation and hygiene. The major part is borne by children in developing countries.

This estimation of the global disease burden caused by unsafe WSH suggests a significant burden of preventable disease attributable to this cause in developing nations, and a non-negligible burden in developed countries. 
The disease burden caused by the risk factor unsafe water, sanitation and hygiene was estimated at the global level in 1990 (Murray and Lopez 1996a). This original estimate examined water, sanitation and hygiene in terms of diarrhoeal and selected parasitic diseases, based on the partial attribution of their disease burden to the risk factor. It was found that worldwide the risk factor accounted for $5.3 \%$ of all deaths and 6.8\% of all disability-adjusted life years (DALYs). Other communicable (e.g. hepatitis A and $\mathrm{E}$, malaria) and noncommunicable diseases (arsenicosis, fluorosis, methaemoglobinaemia) were not considered in that assessment.

\subsection{Sanitation}

Ideally, sanitation (i.e. human excreta management) should result in the isolation or destruction of pathogenic material and, hence, a break in the transmission pathway. In a comprehensive literature review, Esrey et al. (1991) identified 30 studies, from a variety of different countries (including Bangladesh, Brazil, Chile, Guatemala, Kenya, Malaysia and Panama), that examined the impact of sanitation on disease transmission. Twenty-one of those studies reported health improvements (median 22\% reduction in diarrhoea morbidity), with a greater median reduction being seen in the rigorous studies (36\% reduction). Several studies have isolated various faecal-oral pathogens from the faeces of sick people and the transmission of such pathogens isolated from infected faeces to human hosts has been shown in numerous studies (e.g. for Shigella [Dupont et al. 1989]). Clearly, the relationship is both plausible and coherent. Indiscriminate refuse disposal and poor drainage systems has also been a disturbing issue in terms of sanitation.

\subsection{Water}

The number of outbreaks of infectious diarrhoea caused by faecal-oral pathogens in developed countries attests to the efficiency of this mode of transmission. In the United States, for example, 14 outbreaks of infectious etiology associated with drinking water were reported for the two-year period 1997-1998 (Barwick et al. 2000).

In developing countries, it is not only water contaminated at source or during distribution that is an issue, but water stored within the home which may also become contaminated (arguably a hygiene issue). For example, in a literature review, VanDerslice and Briscoe (1993) found 11 observational studies showing that mean coliform levels (an indicator of contamination) were considerably higher in household water containers than in the original source waters.

Numerous epidemiological studies and outbreak investigations have found an association between poor water quality and infectious diarrhoea. In France, water that did not meet microbiological standards was associated with an increased risk of gastroenteritis (RR 1.36, CI 1.24-1.49) (Ferley et al. 1986). In the Philippines, Moe et al. (1991) reported an odds ratio (OR) of 1.92 (CI 1.27-2.91) for diarrhoea following consumption of water contaminated with high levels of Escherichia coli (a faecal indicator bacteria). Mahalanabis et al. (1991) reported that children with prolonged diarrhoeal illness (more than 14 days) were more likely to drink water from an unprotected water source (OR 1.56, CI 1.18-2.06). Birmingham et al. (1997) conducted an epidemiological investigation to identify sources of infection and risk factors for cholera in Burundi during an epidemic in 1992. Water from Lake Tanganyika was implicated, as a case control study found that both bathing in the lake (OR 1.6, CI 1.1-2.1) and drinking its water (OR 2.78, CI 1.0-7.5) were independently related to illness; additionally, Vibrio cholerae was isolated from the lake water.

The causal relationship between ingesting water of poor sanitary quality and diarrhoeal illness has been observed worldwide, using a variety of techniques and assessing quality in a number of different ways. The biological gradient can be illustrated by increases in infectious diarrhoea morbidity as contamination levels increase, and also as consumption of water from a single contaminated source increases. For example, Njemanze et al. (1999) examined the annual diarrhoeal incidence rate (per 1000 population) in 39 communities in Imo State, Nigeria, in relation to the characteristics (including pollution) of their drinking water source. Sources were classified from A to $\mathrm{C}$ with A representing the most desirable sources (with favourable geology, sparse population and clean and unpolluted water). Diarrhoeal incidence rate was found to show a statistically significant increase with a mean of 1.61 for category A, a mean of 6.25 for category $\mathrm{B}$, and a mean of 15.6 for category $\mathrm{C}$.

The relationship between infectious diarrhoea and transmission of pathogens through water is both plausible and coherent. Isolation and enumeration of specific pathogens in water are often not feasible 
or very imprecise; thus a more common measure of faecal contamination is derived from the use of indicator bacteria. There have been many studies using such indicator species that have demonstrated the faecal contam- ination of drinking water sources in both developed and developing countries (e.g. Ampofo 1997).

\subsection{Hygiene}

A number of studies have attempted to examine the role of personal and domestic hygiene, although in many cases some of the "hygiene" measures or interventions could also impact on sanitation, and hygiene interventions may also interact with water quality.

Six studies examined by Esrey et al. (1991) identified reductions in diarrhoea morbidity associated with the uptake of hygiene interventions. These ranged from $14 \%$ to $48 \%$, with a median reduction of $33 \%$. In a more recent review, Huttly et al. (1997) identified a further four studies addressing the impact of improved hygiene. All four studies showed a decrease in diarrhoea, as did a subsequent study of Curtis et al. (2000). These studies were conducted in diverse locations including Bangladesh, Burma, Guatemala and the United States.

The temporal adoption of hygiene measures can be illustrated by the study by Ahmed et al. (1993). This group compared cleanliness and diarrhoea levels in villages with and without hygiene education interventions. Higher adoption rates of the intervention were associated with a better cleanliness state, which was paralleled by a decrease in diarrhoea and malnutrition rates. These differences were found to increase over time as more villagers adopted the intervention.

Alam et al. (1989) studied the effect of four different hygiene mea- sures (source of washing water; presence of faeces in the yard; hand washing before serving food; and hand washing after defecation). They showed decreasing diarrhoea incidence as the number of adopted hygienic practices increased (4.9 cases per child-year for one practice to 2.6 cases for all four).

A review by Feachem (1984) documented the presence of pathogens on the hands following toilet activities. In the same review, Feachem also noted a number of studies on hand washing which demonstrated the almost complete removal (98-100\%) of seeded bacteria.

\section{THEORETICAL FRAMEWORK}

The epidemiological transition model, developed by Omran, focuses on the complex changes in the patterns of health and disease; the interaction between these patterns and their demographic, economic, sociological determinants and consequence. In its original form, the model proposed three stages referred as Age of pestilence and famine; was characterized by a demographic regime of high and fluctuating birth and death rate that reflected Old World epidemics of infection and famine.

The third stage of Omran's transition constituted the "Age of degenerative and Man-made diseases". These stage of transition was largely driven by social factors; such as lifestyle, diet, occupation and income. Omran, argued that, as infectious and parasitic diseases receded, their place would be taken by a series of chronic, degenerative disease associated with ageing populations such as cardiovascular diseases, stroke and cancers: These diseases will become significant causes of mortality. More recently, two more stages have been added (the fourth and the fifth stage).

The fifth stage referred to as "Age of emergent and re-emergent infections". This is characterized by the resurgence of both old and new infectious and parasitic diseases. These stage, proposed in Omran's original and revised models occur sequentially. eg; Malaria, Cholera, Meningitis in Ghana, TB in Africa/worldwide.

\section{Methodology}

- Retrospective review and analysis of records of patients at the TTH, Central and West Hospitals.

- Secondary data from the Ghana Statistical Service of Tamale were collected and analyzed to explain the health and demographic development of the population from 1970 to 2010.

- Records of registered demographic and diagnosis of characteristics of patients were taken from the physician's specialist clinic, chest clinic, diabetic clinic, death certificate books from the medical wards, mortuary, Laboratories and the births and deaths registration center.

- Ethical Clarence was obtained from the research directorate of the TTH. 


\section{DISCUSSION OF FINDINGS}

Table1. Population Census of Northern Region Since 1960

\begin{tabular}{|l|l|l|c|c|}
\hline Year & Population & \% Increase & \% Share Of Ghana's Population & Intecensal Growth Rate \\
\hline 1960 & 531573 & - & 7.9 & - \\
\hline 1970 & 727618 & 36.9 & 8.5 & 3.2 \\
\hline 1984 & 1164583 & 60.1 & 9.5 & 3.4 \\
\hline 2000 & 182802 & 56.3 & 9.6 & 2.8 \\
\hline 2010 & 2479461 & 36.2 & 10.1 & 2.9 \\
\hline
\end{tabular}

Source: Ghana Statistical Service (Tamale)

From the above table rapid population growth coupled with unplanned urbanization leads to pressure on the land which leads to inadequate space for social amenities and infrastructure which also leads to pressure on fewer facilities like public toilets, indiscriminate disposal of refuse including lack toilet facilities and refuse damping sites in most homes and communities.

Table2. Morbidity of Infectious Diseases at the Three Main Hospitals of Tamale Metropolitan Area (Tth, Central and West Hospitals Second Quaterly Review- 2017

\begin{tabular}{|l|l|l|}
\hline \multicolumn{1}{|c|}{ Name Of Disease } & \multicolumn{1}{c|}{ Number of Patients } & \multicolumn{1}{c|}{ Percentage (\%) } \\
\hline 1. Malaria & 3000 & 43.9 \\
\hline 2. Gastroenterities & 750 & 10.98 \\
\hline 3. Typhoid Fever & 430 & 6.29 \\
\hline 4.Uti & 400 & 5.85 \\
\hline 5.Diarrhoea & 350 & 5.12 \\
\hline 6.Hiv/Ptb & 360 & 5.27 \\
\hline 7.Dicentry & 480 & 7.02 \\
\hline 8.Worm Infestation & 180 & 2.63 \\
\hline 9.Hepatitis & 200 & 2.92 \\
\hline 10.Anaemia & 680 & 9.95 \\
\hline Total & 6830 & 100 \\
\hline
\end{tabular}

Table3. Review of Records of Patients at the Microbiology Laboratories of the Three Main Hospitals in the Tamale Metroplitan Area (Second Quaterly Review)-2017

\begin{tabular}{|l|l|l|}
\hline \multicolumn{1}{|c|}{ Name of Disease } & \multicolumn{1}{c|}{ Number } & Percentage \\
\hline 1.Plasmodium (Malaria) & 750 & 22.05 \\
\hline 2.Ecoli (Uti) & 700 & 17.64 \\
\hline 3.Salmonela (Decentry) & 650 & 19.11 \\
\hline 4.V. Cholera (Diarrhoea) & 650 & 19.11 \\
\hline 5.Afb (Ptb) & 500 & 14.70 \\
\hline 6.Taenia (Hook Worm) & 50 & 1.47 \\
\hline 7.Schistosoma (Bilhazhia) & 150 & 4.41 \\
\hline 8.Klebsiela (Pneumonia) & 10 & 0.29 \\
\hline 9.Morasella (Resistant/ Urti) & 10 & 0.29 \\
\hline 10.HEPATITIS (Hbsag) & 30 & 0.26 \\
\hline Total & 3400 & 100 \\
\hline
\end{tabular}

As seen in the tables above, the second quarter review periods including the months of April, May, June and July. These are the months of rainy season in the northern Ghana of this periods seasonal epidemics are usually recorded as malaria, gastro enteric disease and water borne diseases (Ghana Health Service, 1994). This is confirmed as documented in the records of laboratory and OPD attendees of the three major hospitals of the tamale metropolitan area in 2017.

Plasmodium falciparium, the causative agent of malaria, topping the list in the laboratories could be explained by the fact that the environment is very favorable for the development of mosquitos which are the primary host of this protozoa. Stagnant water bodies as a result of choked gutters, empty containers and indiscriminate disposal of refuse. These are very good conditions for fast development of mosquitos.

Of all the pollutants, human excreta (faeces) are the most dangerous to health. One gram of fresh faeces from an infected person can contain around $10^{6}$ viral pathogens, $10^{6}-10^{8}$ bacterial pathogens, $10^{4}$ protozoan cysts or oocysts, and $10-10^{4}$ helminth eggs. This explains the importance of particular 
interventions, notably the safe disposal of faeces, in preventing disease transmission. Apart from that incriminate open defecation explains the high number of enteric and Uneurobic bacteria documented in the laboratory records. These bacteria include Ecoli, vibro cholera, Salmonella, Enterica and Semolina typhoid, Amoebae, Taenia, Solium and T saginata, schistosomia, hookworm. Defication openly by animals and human beings could be washed into the waters and spread the above named bacteria. Also flies which are normally increased during this period sit on faecal matter and carry them to our homes and drop them in our food. Whether contaminated water or food by this micro will lead to disease of gatro enteric colitis. This is confirmed as shown in the OPD results in the three hospitals.

Apart from the micro biology infestation, inorganic substances could be washed along into our water bodies. These are normally produced by exhaust gases produced from motor bikes and cars. The polluted chemicals include carbon monoxide, mercury, nitric acid, cyanide etc. these substances when taken into our bodies causes cancer of the liver, prostate, kidney, colon and other organs of the body.

all the above pollutants can cause diseases that can cause our death or reduce our abilities to function. When one falls sick of malaria, cholera, cancer etc one cannot go to work. This situation leads to poverty, failure in economic and developmental endeavors. The pictures below throw more light on the above discussion.

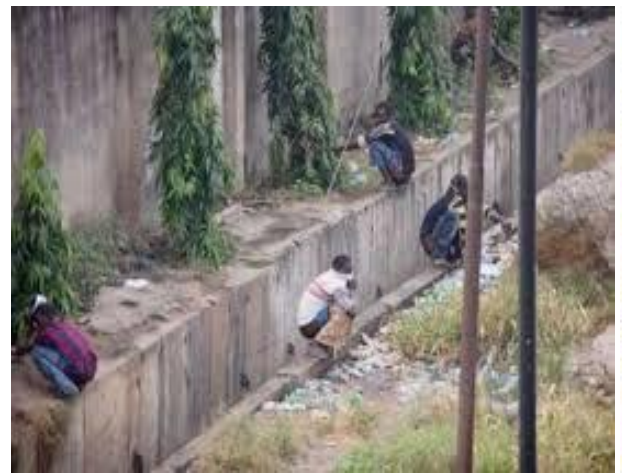

Fig1

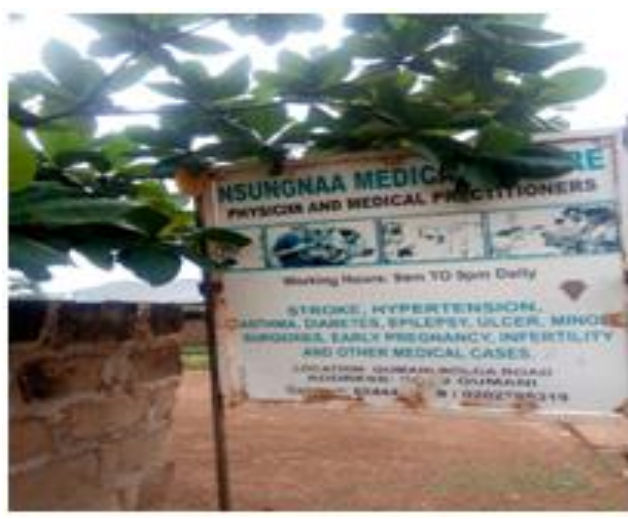

Fig3

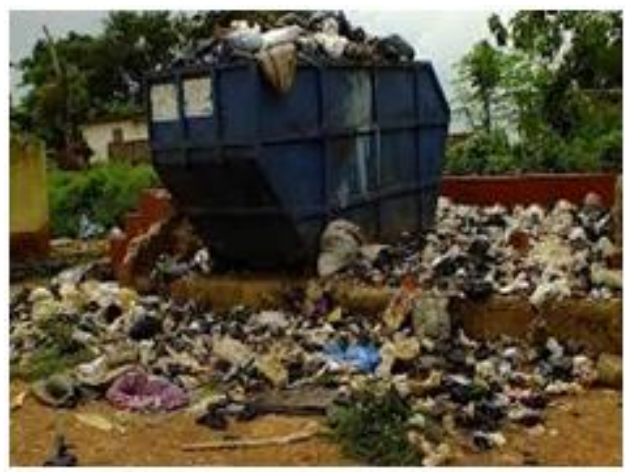

Fig2

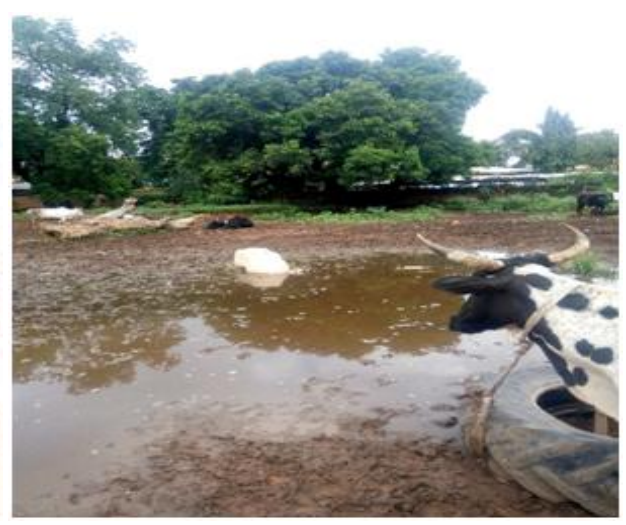

Fig4

\section{CONCLUSION}

Seasonal epidemics, fueled by the inhabitants of the Tamale Metropolitan area, goes in line with the third and fifth theories of the Omran epidemiological transition; reappearance of infectious diseases and the double disease burden. As found in the second quarterly review records in the hospitals. Most of the diseases documented were caused by microbes as a results of lack of sanitation and environmental pollution. Ecoli, vibron cholera, salmonella, entarica and salmonella typhoid are all enteric organisms which come out of human and animal faeces. Careless and open defecations are the causes of rise in prevalence of diarrhea diseases typhoid and gastroenteritis. As shown in the records, Malaria is still the top cause of morbidity in all the three hospitals, that means the environmental conditions are very favorable for the development of plasmodium falciparium species; the etiology of malaria. The problem is that when one gets sick of any of these diseases, the person cannot go to work for at least, three days. Time is lost, productivity is reduced and poverty is enhanced (WHO,2013) 


\section{RECOMMENDATION}

- The ministry of health has to ensure that primary health approach is adopted by all health facilities and health institutions (the strategy for achieving the Millennium Development Goals, WHO 1979).

- All Ghanaians have to be educated on the killer diseases so as to achieve perfect health for all (MDG 3).

- Hygiene; prevention of disease will have to be taught at all levels of schools in the country (MDG 2).

- Reintroduction of sanitary inspectors is very needful especially in the urban slums and rural communities.

- Refuse collection agencies in the urban residential areas like Zoomlion Ghana etc should be encouraged.

- More vibrant metropolitan assembly and town and country planning is necessary.

- National health institutions should acknowledge and address the impact of sanitation on the global disease burden, the contribution of improved sanitation to reducing that disease burden and the potential benefits for public health outcomes.

- International donors should prioritise support for programmes in countries with low sanitation coverage and high burden of sanitation-related disease and invest in research and evaluation to understand the relative health impacts and effects of sanitation intervention.

- Ghana government should ensure that sanitation is addressed within all relevant health policies, regulations, guidelines and procedures and establish targets and indicators for monitoring improvements in sanitation related diseases.

- Government should strengthen public health legal and regulatory frameworks to improve intersectoral coordination between ministries and agencies responsible for sanitation at different levels and enhance accountability for results.

- National and sub-national health programme priorities should take account of sanitation-related disease burden and ensure that sanitation and hygiene are fully integrated within disease specific and national health programmes.

\section{ACKNOWLEDGMENT}

My sincere acknowledgement goes to the Tamale Teaching Hospital for allowing me use the clinical and the laboratory results to support my research findings. this is evidence based approach. And to Mr. Doklah Kwame Anthony of Catholic University, ICST Department for helping with typesetting and the editorial work. He served me as my personal Research Assistant

\section{REFERENCES}

[1] David Prado (2017). Global perspectives: local insight in managing infection. Inspiration.

[2] Omran A. (1974). Epidemiologyical transition.

[3] WHO (2013). Economic summit. WHO Annual bulletin

\section{AUTHOR'S BIOGRAPHY}

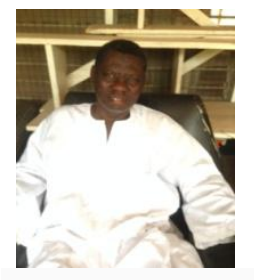

Dr. Abdallah Iddrisu Yahaya is a Ghanaian by Nationalaity. He is a senior lecturer at University for development studies (UDS) at the department of internal medicine. he is a consultant Physcian at the Tamale Teaching Hospital, TTH. he is also the Regional TB clinician for Northern Ghana. Medical Doctor, MSC community Health and BSc. Ziology and PHD candidate, Millar Open University.

Citation: Dr. Abdallah Iddrisu Yahaya. "Increase in Infectious Diseases as a Result of Poor Environmental Sanitation in the Tamale Metropolis". International Journal of Humanities Social Sciences and Education (IJHSSE), vol 5, no. 6, 2018, pp. 58-65. doi: http://dx.doi.org/10.20431/2349-0381.0506008.

Copyright: () 2018 Authors. This is an open-access article distributed under the terms of the Creative Commons Attribution License, which permits unrestricted use, distribution, and reproduction in any medium, provided the original author and source are credited. 